\title{
All about alleles
}

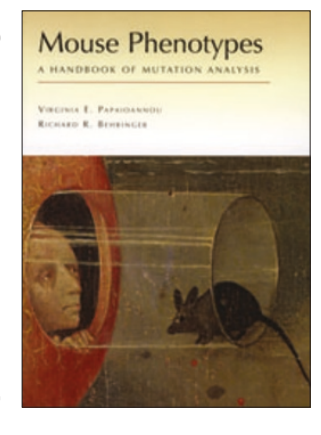

\section{Mouse Phenotypes: A Handbook of Mutation Analysis}

\section{Virginia E. Papaioannou \& Richard R. Behringer}

Cold Spring Harbor Laboratory Press, 2005 $235 \mathrm{pp}$. hardcover, $\$ 80$ ISBN 0879696400

\section{Reviewed by Maja Bucan}

Developmental biologists commonly study genes or pathways that have similar functions in a wide range of model organisms. Each of these model organisms has a unique set of features and preferred experimental approaches. In the mouse, most available mutant alleles have been generated by manipulation of embryonic stem cells and transgenesis. Among these, strategies for generating alleles with conditional gene expression are particularly powerful for dissecting multiple gene functions. But the true value of a mutant comes from its phenotypic analysis, especially when this analysis combines molecular, genetic and cell biological approaches. The book Mouse Phenotypes: A Handbook of Mutation Analysis by Virginia Papaioannou and Richard Behringer focuses on both aspects of mouse (2) genetics - mutant generation and analysis.

Mouse Phenotypes conveys the relative advantages and disadvantages of the mouse as a reference organism for developmental biology. The eight chapters cover topics ranging from how to generate mutants to how to analyze different classes of phenotypes in embryos and in adult mice. The last chapter, titled "Getting Around an Early Lethal Phenotype," outlines several approaches that can be used to study the function of any gene that has a complex temporal and spatial expression pattern. These approaches range from classical methods, such as the generation of chimeric mice, to strategies based on conditional regulation of gene expression. In addition to a simple but detailed description of embryological procedures, the book provides an excellent guide to the genetic and molecular analysis of mouse mutants. I benefited tremendously from reading the book from cover to cover and recommend that students and postdocs do the same, especially because of the material presented in boxes and 'helpful hints'. For readers who are in a rush or wish to focus on specific aspects of the analysis that are relevant to a mutant of interest, the outline of 'dichotomous branching pathway to the analysis' (e.g. " if you observe this phenotype, go to this section of the book") suggests the most appropriate section.

This book focuses primarily on the analysis of mutants generated by targeted mutagenesis and emphasizes the importance of knowing the

Maja Bucan is in the Department of Genetics, University of Pennsylvania School of Medicine, Philadelphia, Pennsylvania 19104, USA.

email:bucan@pobox.upenn.edu expression pattern of a gene when designing experiments and analyzing mutant phenotypes. The book is equally helpful, however, in the absence of knowledge about the expression pattern or before the molecular identification of the disrupted gene, for example, in mutants identified in forward genetic screens.

The figures, schematics and drawings throughout the book are informative and nicely illustrate important aspects of mouse genetics. This is particularly true for examples of representative mouse mutants on color plates selected from publications by the authors and their colleagues.

Most interesting to me was a diverse set of experimental procedures suggested for the analysis of mutant phenotypes at the peri-implantation and early post-implantation stages. Dissecting early-stage mouse embryos can be very difficult, even for those who are technically competent. It is generally believed that this skill can be acquired only from an experienced colleague or advisor, who learned from his or her advisor. I admire the authors' attempts to overcome this myth by suggesting a variety of procedures to observe mutant phenotypes in dissected or cultured embryos. Although visualization of defined embryonic structures by in situ hybridization using known developmental markers helps in the depiction of dynamic morphological changes in a three-dimensional space, the authors do not convey the high level of skill and patience needed to find the embryo proper, to keep embryos intact during in situ hybridization and to produce publishable results.

Mouse Phenotypes will find a place on the bookshelves of many mouse geneticists and developmental biologists, next to The Atlas of Mouse Development by Kaufman and Manipulating the Mouse Embryo by Nagy et al., which focus primarily on the analysis of normal phenotypes. Papaioannou and Behringer worked to avoid overlap with the latter and point the reader to experimental procedures covered by Nagy and colleagues when appropriate.

In a quickly developing field with many new functional genomics resources, it is always possible to find approaches that are missed or better covered in a recent review article. For example, this book does not include references to several new databases that have large-scale microarray and in situ expression data, which may turn out to be useful for the interpretation of unexpected mutant phenotypes or in the search for a tissue-specific promoter. Also, the section on phenotypic analysis of embryos could have included recently developed imaging techniques and a much longer list of experimental procedures for the analysis of phenotypes in the adult mouse. For example, in-depth biochemical and physiological analysis of a newly identified mutant may uncover subtle phenotypes or explain overt visible or behavioral phenotypes. Furthermore, molecular phenotypes such as those identified by microarray analysis may provide insight, if not into the primary defect, then into the secondary phenotypes that may be particularly important in comparisons to human disease. Altogether, the title of the book implies a more systematic and comprehensive collection of methods than is described in the book. This comment should not be taken as a criticism but rather as an encouragement to the authors to start planning the second volume or edition. In the meantime, I hope to persuade you to read this handbook before you attempt to analyze your next mouse mutant, regardless of the method used to generate it. 